SUBJECT AREAS:

BREAST CANCER

TRANSLATIONAL RESEARCH

Received

27 December 2013

Accepted

2 June 2014

Published

19 June 2014

Correspondence and requests for materials should be addressed to G.J.Z. Iguoj_zhang@ yahoo.com)

* These authors contributed equally to this work.

$\uparrow$ Current address:

Breast Tumor Center,

Chancheng District

Central Hospital of

Foshan City, Foshan

528031, China.

\section{Clinicopathological features and prognostic factors of young breast cancers in Eastern Guangdong of China}

\author{
Jin-Tao Wei ${ }^{1 * \dagger}$, Wen-He Huang ${ }^{1 *}$, Cai-Wen Du², Si-Qi Qiu', Xiao-Long Wei ${ }^{3}$, Jing Liv ${ }^{3}$ \\ \& Guo-Jun Zhang',
}

\begin{abstract}
'The Breast Center, Cancer Hospital of Shantou University Medical College, Shantou 515041, China, ${ }^{2}$ Department of Breast Medical Oncology, Cancer Hospital of Shantou University Medical College, Shantou 51504 1, China, ${ }^{3}$ Cancer Research Center, Shantou University Medical College, Shantou 515041, China.
\end{abstract}

Breast cancer in young women is typically with higher proportion of adverse pathological features. Breast cancer with BRCA1 mutation is often early-onset, and is usually associated with triple negative phenotpe. In this study, we aim to analyze the clinicopathological characteristics and prognosis in young breast cancer patients ( $\leq 35$ years old) comparing to non-young patients ( $>35$ years old). A total of 1913 cases of primary breast carcinoma with stage I-III were enrolled, with 283 cases diagnosed as young patients. No significant difference was observed in tumor size, TNM staging, lymph node metastasis, ER, HER-2 or histological grade between young and non-young patients. Multivariate analysis demonstrated that age was an independent prognostic factor for overall survival (OS). In 70 samples of young patients available, BRCA1 was immunohistochemically positive $85.7 \%$ in cytoplasm and $41.4 \%$ in nuclear. BRCA1 nuclear expression is not significantly associated with clinicopathological characteristics in young breast cancer patients.

reast cancer has become the most common cancer in women globally ${ }^{1}$. Patients younger than 40 or 35 years old at diagnosis are usually considered as a young population ${ }^{2}$. The incidence of breast cancer is as low as $0.50 \%$ in female younger than 39 years old, while $3.78 \%$ in female aged $40-59$ years old, published data indicated that death rate is almost the same in two age groups ${ }^{1}$. Although young patients with breast cancers were thought to be associated with poor prognosis, the reason is not well defined. Several reports suggested that many factors such as the adverse pathological features ${ }^{3-5}$ and delays in diagnosis ${ }^{6-8}$, as well as lacking reliable screening methods in young women, probably indicate the poor prognosis in such group of patients.

Previous studies have tried to determine potential biological characteristics of breast cancer in young women. Young breast cancer patients were shown to have biologically more ER negative ${ }^{9-12}$ and higher histological grade, more triple-negative subtype ${ }^{13,14}$, as well as more extensive intraductal component (EIC) ${ }^{12}$ and fewer ductal carcinoma in $\mathrm{situ}^{15}$. In general, young women seem like to have tumors with more aggressive biological characteristics, after adjusting these factors, age is still an independent factor for prognosis in several studies ${ }^{16,17}$. Survival analysis also demonstrated that young breast cancer patients have early recurrence with shorter disease free survival (DFS) and OS. These findings suggest young age in breast cancers is a poor prognostic factor and the patients with young age should be treated with more intense therapeutic strategies.

However, several randomized clinical trials showed similar survival rates after breast conserving therapy followed by radiotherapy and mastectomy in early breast cancer. But the local recurrence after breast conserving therapy can be up to $30 \%$ for young patients ${ }^{18,19}$. Even followed by radiotherapy, rate of local recurrence is still higher for young patients when comparing with older patients, but data of which is different among studies in mastectomy group ${ }^{20-24}$.

BRCA1 is a tumor suppressor gene, which plays an important role in maintaining genomic integrity by protecting cells from double-strand breaks (DSB) that arise during DNA replication or after DNA damage ${ }^{25}$. BRCA1-mutated associated breast cancer is often early-onset, highly prevalent between 30 and 50 years of age. And they are mostly ER negative and HER-2 negative ${ }^{26}$. It has some similarities with young breast cancer while only a fraction of young women carries BRCA mutations ${ }^{27,28}$.

The study aims to investigate the clinicopathological characteristics and the role of BRCA1 protein in young Chinese breast cancer patients in Eastern Guang-Dong area. We also analyzed the recurrence and survival to identify the prognostic factors in this group of patients. 


\section{Results}

The median age at diagnosis was 47 years (range $18-69$ years). The patient was divided into two groups according to age factor (i.e., $\leq 35$ years old vs. $>35$ years old). The distribution of clinicopathological features in two groups was shown in Table 1. A higher proportion of family history of breast cancer with first-degree relatives was observed in non-young patients $(2.6 \%$ vs. $0.7 \%, \mathrm{P}=0.048)$. In young patients, $72.4 \%$ of them are with tumor being less than $5 \mathrm{~cm}$ in diameter, which has no difference in comparison to $76.1 \%$ in nonyoung patients. Similarly, there are no differences in lymph node status, ER status, HER-2 status, and histological grade between two age groups. Approximately $67 \sim 73 \%$ of breast cancers were luminal subtype in both group, and $49 \sim 57 \%$ of them were with histological grade 2 .

Of 70 cases of young breast cancer patients, 29 cases (41.4\%) were nuclear positive for BRCA1 IHC staining as shown in Figure 1. There were no differences observed in histological grade, ER expression, or HER-2 expression, and distal metastasis associated with BRCA1 protein nuclear expression shown in Table 2. However, there seemed to have a trend that the positivity of BRCA1 nuclear expression is reduced in the patients with larger tumor size, positive lymph node or higher histological grade.

Univariate and multivariate analysis were used to analyze the prognostic factors of overall survival (OS) for patients. In Table 3 and Figure 2, univariate analysis showed that young age ( $\leq 35$ years old), larger tumor size, more axillary lymph nodes involvement, ER negative were significantly associated with poorer OS. The family history of breast cancer with first-degree relatives was not significantly associated with OS. Table 3 presents results of Cox's proportional hazards analysis for OS. In addition to T factor of TNM stage, lymph node status, ER status and young age ( $\leq 35$ years old) $(\mathrm{HR}=$ $1.489, \mathrm{P}=0.006$ ) were all independent prognostic factors for poorer OS in multivariate analysis.

We also applied multivariate analyses to address factors that may influence the OS in the young breast cancer patients. Table 4 shows univariate and multivariate analysis of factors associated with OS for the patients aged $\leq 35$ years old. BRCA1 protein nuclear expression was not associated with OS in young breast cancer patients. Large tumor size, more axillary lymph nodes involvement and ER negativity were independent factors for poorer OS.

\section{Discussion}

Previous report indicated that young age was an independent prognosis factor for relapse and death in breast cancer patients ${ }^{16}$. The mechanism underlying poor prognosis for young breast cancer was still undefined. As reported previously, adverse or aggressive biological features might be associated with young breast cancer patients. The subtype classification defined by IHC on ER and/or PR, HER2 and Ki-67 were used widely to predict the prognosis of breast cancer patients, which also provided some clues for making therapeutic strategies. So, will be there any specific biomarkers for prognosis in young breast cancer patients?

In comparison to a higher proportion of family history in young breast cancer patients than older patients ( $24 \%$ vs. $17 \%)$ reported by Sidoni et al. ${ }^{4}$, the present study found that only 2 of 283 young patients $(0.7 \%)$ had family history of breast cancer within first relatives. The low incidence of family history is probably because of small number of cohorts. Xiong et al. ${ }^{3}$ reported that $44 \%$ of young patients presented with locally advanced or stage IV disease, having a higher proportion of later stage than older patients. In our study, tumor size or lymph node status didn't vary between young and non-young groups. That may be associated with better awareness and screening in foreign countries so it is more common to find early breast cancer when the patients still were asymptomatic. While in China, in particular in Eastern Guangdong, a relatively poor suburban/urban area, most women went to hospital when they found palpable abnormality in breast. Our data showed that in which were nearly consistent with previous reports ${ }^{4,9,14}$. In the Eastern Guang-Dong area, no differences were not observed in terms of histological grade, ER status and HER2 expression between young and non-young groups in our study. The relatively high percentage of HER-2 expression is probably due to

Table 1 | Clinico-pathological characteristics by age at diagnosis in breast cancer patients

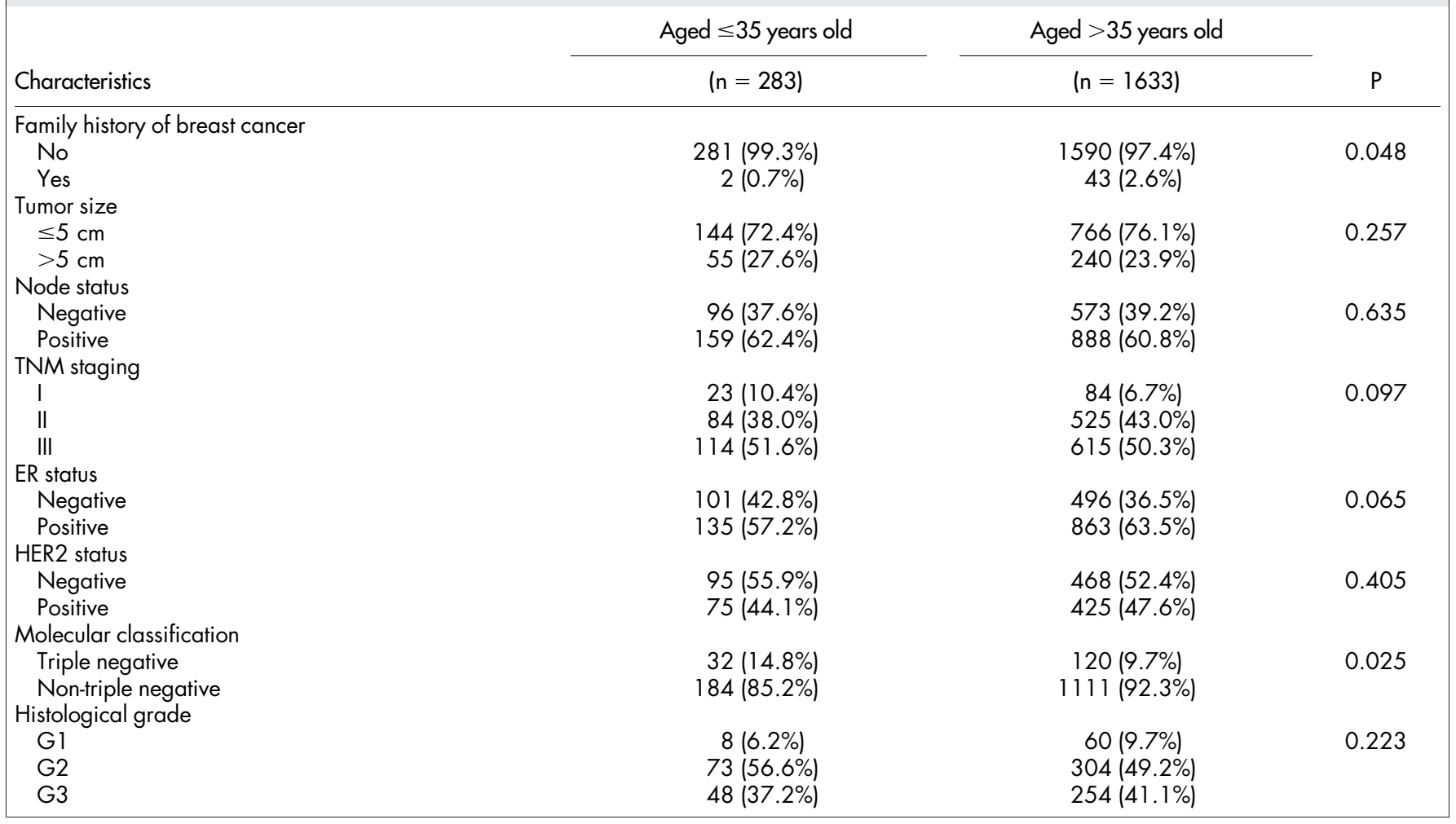



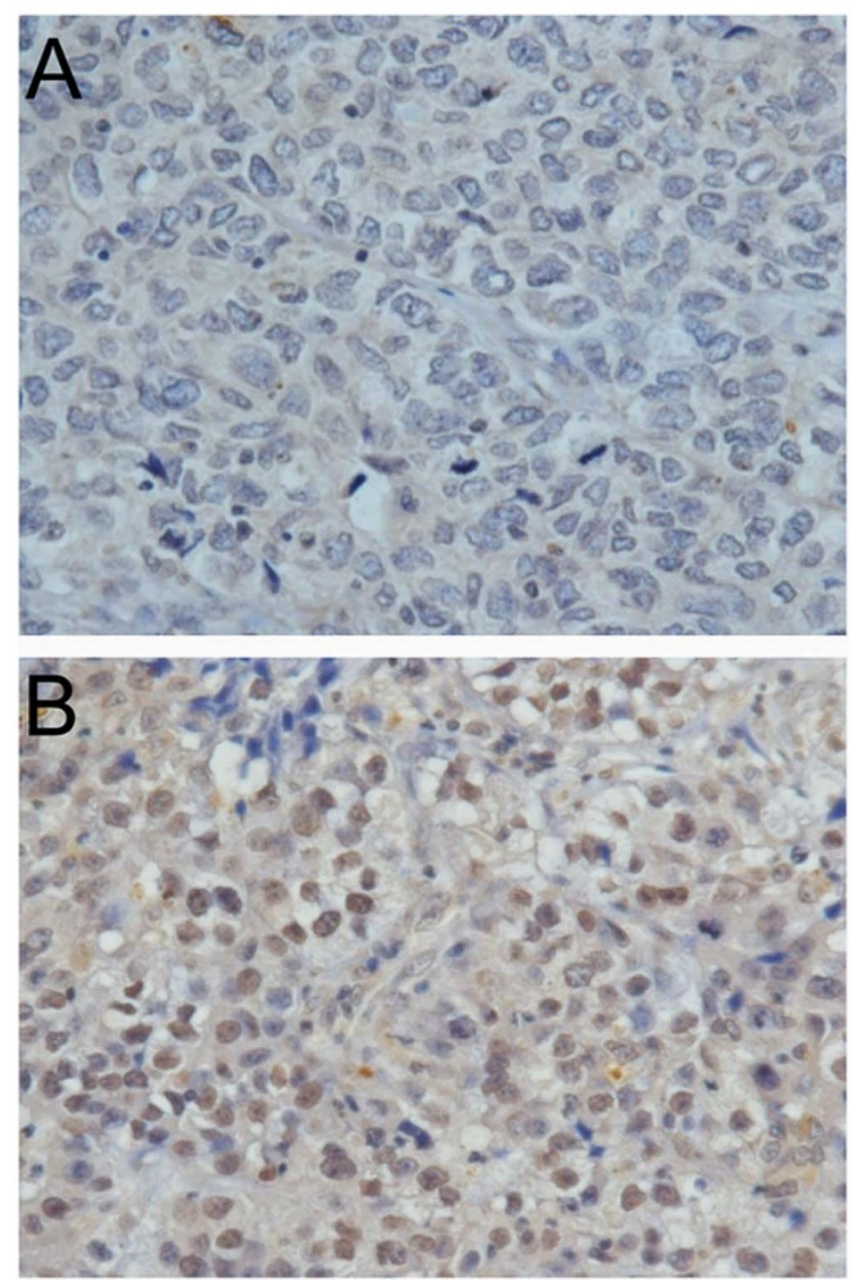

Figure 1 BRCA1 nuclear expression in young breast cancer patients $(400 \times)$. (a) negative BRCA1 nuclear staining, and (b) positive BRCA1 nuclear staining. almost $30 \%$ of the paraffin-embedded tissue sections are missing, in which HER-2 status remained unknown or uncertain.

BRCA1, a tumor suppressor gene, encodes a $220 \mathrm{KDa}$ protein to function in the response for double-strand breaks (DSBs) by homologous recombination (HR). BRCA1 mutation cause functional defect in HR-mediated repair ${ }^{29}$, and lose function to inhibit tumorigenesis. Although BRCA1 mutation is rare in sporadic breast cancer, but reduction of BRCA1 nuclear expression in breast cancer tissue is common $^{30}$. Xiang et al. ${ }^{31}$ found that $69 \%$ of cases were negative for BRCA1 IHC staining in 101 breast cancer patients, and they also had higher Ki-67 expression. Rakha et al. ${ }^{30}$ found that absent or reduced nuclear BRCA1 expression was observed in $80 \%$ of basal-like breast cancer patients. The reduced BRCA1 expression was also associated with high-grade, advanced lymph node stage, larger tumor size, vascular invasion, negative estrogen receptor and/or progesterone receptor, androgen receptor expression, and positive p53 expression. Bogdani et al. ${ }^{32}$ found that absent BRCA1 nuclear staining was more frequently found in young women than older patients. Absent or reduced nuclear BRCA1 expression seems to be predictor for poor prognosis.

In this study, we aimed to better understand BRCA1 protein's value to predict prognoses in young breast cancer patients. As BRCA1 protein mainly repairs DNA when they get damaged, we attempted to detect the nuclear expression of BRCA1 protein immunohistochemically. Kim et al. ${ }^{33}$ found that $58.7 \%$ of the breast cancer cases were BRCA1 negative in cytoplasma and nuclear positive rate were $24 . \%$. There were no differences in either nuclear or cytoplasmic expression among different molecular subtypes. It was not significantly associated with disease-free survival or overall survival in their study. The rate of BRCA1 positive from other reports ranged from $31 \%$ to $65.7 \%{ }^{30,31,34-36}$. In those studies, absent or reduced nuclear BRCA1 expression was associated with lager tumor size, greater axillary lymph node metastasis, higher histological grade, vascular invasion, negative estrogen receptor, higher rate of $\mathrm{Ki}-67$ positive, more frequently in triple negative subtype. In contrast, in our study, there seemed a trend that reduced BRCA1 expression was associated with larger tumor size, positive lymph nodes involved and higher histological grade, but they did not reach statistical significance. BRCA1 nuclear expression was not associated with overall survival

\begin{tabular}{|c|c|c|c|}
\hline Characteristics & BRCA1 nuclear negative & BRCAI nuclear positive & $P$ \\
\hline \multicolumn{4}{|l|}{ Tumor size } \\
\hline $\mathrm{T} 1 / \mathrm{T} 2$ & $21(53.8 \%)$ & $18(46.2 \%)$ & \multirow[t]{2}{*}{0.147} \\
\hline $\mathrm{T} 3 / \mathrm{T} 4$ & $14(73.7 \%)$ & $5(26.3 \%)$ & \\
\hline \multicolumn{4}{|l|}{ Node status } \\
\hline Negative & $16(57.1 \%)$ & $12(42.9 \%)$ & \multirow[t]{2}{*}{0.843} \\
\hline Positive & $25(59.5 \%)$ & $17(40.5 \%)$ & \\
\hline \multicolumn{4}{|l|}{ Distal metastasis } \\
\hline Negative & $30(57.7 \%)$ & $22(42.3 \%)$ & \multirow[t]{2}{*}{0.800} \\
\hline Positive & $11(61.1 \%)$ & $7(38.9 \%)$ & \\
\hline \multicolumn{4}{|l|}{ TNM stage } \\
\hline I/II & $18(60.0 \%)$ & $12(40.0 \%)$ & \multirow{2}{*}{0.698} \\
\hline III & $22(64.7 \%)$ & $12(35.3 \%)$ & \\
\hline \multicolumn{4}{|c|}{ Histological grade } \\
\hline $\mathrm{G} 1 / \mathrm{G} 2$ & $20(52.6 \%)$ & $18(47.4 \%)$ & \multirow[t]{2}{*}{0.100} \\
\hline G3 & $19(73.1 \%)$ & $7(26.9 \%)$ & \\
\hline \multicolumn{4}{|l|}{ ER status } \\
\hline Negative & $14(58.3 \%)$ & $10(41.7 \%)$ & \multirow[t]{2}{*}{0.977} \\
\hline Positive & $27(58.7 \%)$ & $19(41.3 \%)$ & \\
\hline \multicolumn{4}{|l|}{ HER2 status } \\
\hline Negative & $18(62.1 \%)$ & $11(37.9 \%)$ & \multirow[t]{2}{*}{0.644} \\
\hline Positive & $18(56.3 \%)$ & $14(43.8 \%)$ & \\
\hline \multicolumn{4}{|c|}{ Molecular classification } \\
\hline Non-Luminal & $14(66.7 \%)$ & $7(33.3 \%)$ & \multirow[t]{2}{*}{0.368} \\
\hline Luminal & $27(55.1 \%)$ & $22(44.9 \%)$ & \\
\hline
\end{tabular}


Table 3 | Univariate and multivariate analysis of overall survival in breast cancer patients

Univariate analysis

Multivariate analysis

\begin{tabular}{|c|c|c|c|c|}
\hline Covariates & $\mathrm{HR}(95 \% \mathrm{Cl})$ & $P$ & $\mathrm{HR}(95 \% \mathrm{Cl})$ & $P$ \\
\hline$>35$ & 1.000 & & 1.000 & \\
\hline \multicolumn{5}{|c|}{ Family history of breast cancer } \\
\hline No & 1.000 & & - & \\
\hline Yes & $0.738(0.395-1.379)$ & 0.341 & & \\
\hline \multicolumn{5}{|l|}{ Tumor size } \\
\hline T3 & 5.338 (2.939-9.695) & $<0.001$ & $2.517(1.363-4.647)$ & 0.003 \\
\hline $\mathrm{T} 4$ & $8.728(4.814-15.822)$ & $<0.001$ & 4.208 (2.277-7.776) & $<0.001$ \\
\hline \multicolumn{5}{|l|}{ Node status } \\
\hline Negative & 1.000 & & 1.000 & \\
\hline Positive & 3.655 (2.883-4.633) & $<0.001$ & 4.339 (3.109-6.054) & $<0.001$ \\
\hline \multicolumn{5}{|l|}{ ER status } \\
\hline
\end{tabular}

$(\mathrm{P}=0.311)$. This retrospective study enrolled breast cancer patients during long period spanning for nearly 20 years. The improvement of chemotherapeutic regimes, advancement in surgery technique, changes in TNM staging classification and relevant clinical evidence, although the clinical management was performed according to the guidelines at that time, all the above factors might have influenced on overall survival. And some missing data might also have impact on the clinical outcome we presented in the present study.

In summary, clinicopathological characteristics were not significantly different between breast cancer patients in young age $(\leq 35$ years old) and older age ( $>35$ years old) in the present. Young age was an independent prognosis factor for overall survival in this group of patients. Reduced or lost of nuclear BRCA1 expression in breast cancer tissue seems to be a risk factor for recurrence/metastasis and prognosis.

\section{Methods}

Patients and samples. A total of 1916 primary breast cancer patients were admitted to the Cancer Hospital of Shantou University Medical College between Jan. 1995 to Dec. 2011. The patients with second primary malignant tumors were excluded. The patients with renal or cardiovascular systemic disease were excluded. Patients aged 70-year-old were excluded. All the patients enrolled are women and received surgical treatment. Of them, 283 were diagnosed at younger than 35 years old, and the remaining 1633 cases were older than 36 years old. The TNM staging of breast cancer was based on the American Joint Committee on Cancer (AJCC, $7^{\text {th }}$ edition) criteria.

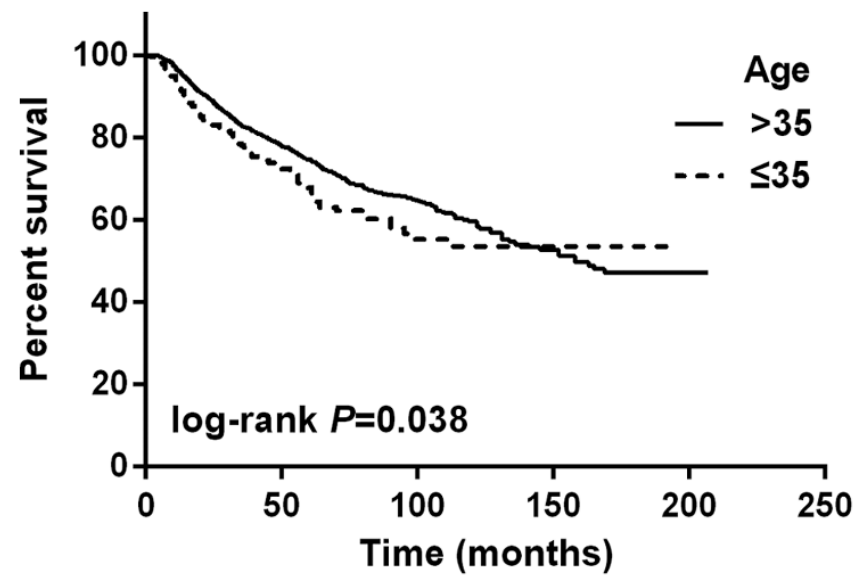

Figure $2 \mid$ Overall survival in young ( $\leq 35$ years old) and non-young $(>35$ years old) breast cancer patients. The log-rank test shows that there is a significant difference between these two survival curves.
Histological grade was categorized as grade I, II or III according to the Nottingham combined histologic grade (Elston-Ellis modification of the Scarff-BloomRichardson grading system) by experienced pathologists. Immunohistochemical subtype classification defined as follows: Luminal A (ER positive and/or PR positive and HER2 negative); Luminal B (ER positive and/or PR positive and HER2 positive); HER2 overexpression (ER negative and PR negative and HER2 positive), Triple negative (ER negative and PR negative and HER2 negative). St. Gallen symposium had redefined the molecular subtype by adding Ki-67. We were still worried for the reliability of assessment of Ki-67 since guidelines for testing of Ki-67 are still lacking. So we preferred to use only ER, PR, HER-2 by IHC to define the molecular subtype.

All patients received surgery after diagnosis. The surgery involved mastectomy or breast conserving surgery combined with axillary lymph node dissection. 1480 cases undergone mastectomy and 87 cases undergone breast conserving surgery. In the young breast cancer group, 240 cases undergone mastectomy and 29 cases undergone breast conserving surgery. Among them, 1565 cases received adjuvant chemotherapy.

Breast cancer tissues from 70 cases of young patients ( $\leq 35$ years old) were available for immunohistochemical analyses. Patients' characteristics and clinical data were extracted based on medical charts and records. All the cases were followed-up until Feb 2013 with a 3-6 months interval for the first 2 years, 6 months interval from 3rd to 5 th year, and one year interval thereafter. DFS was defined as starting from the date of surgery to disease recurrence, and OS was defined as starting from the date of surgery to death or last available date before loss of follow-up, respectively. If a patient's died with other diseases, it would be censored.

Immunohistochemistry (IHC). All immunostainings were performed using formalin-fixed, paraffin-embedded tissue sections. Briefly, $4 \mu \mathrm{m}$ thick sections were obtained by using a microtome, transferred to APES-coated slides, and dried at $60^{\circ} \mathrm{C}$ for 2 hours. Paraffin sections on slides were dewaxed and rehydrated. The slides were then placed in a glass jar with 10/1 mM Tris/EDTA buffer ( $\mathrm{pH} 9.0)$, and heated in a microwave oven for $20 \mathrm{~min}$. The sections were allowed to cool in the jar at room temperature. The slides were then rinsed with phosphate-buffered saline (PBS) and incubated with normal goat serum in PBS (10\%) to block nonspecific staining. Without rinsed, the slides were incubated with primary antibody (BRCA1 MS110, diluted $1: 200$ in PBS, Abcam) at $4{ }^{\circ} \mathrm{C}$ overnight. The endogenous peroxidase activity was blocked by $3 \%$ hydrogen peroxide for $10 \mathrm{~min}$. Immunodetection was performed with Polymer Detection System (Immuno-Bridge ${ }^{+}, \mathrm{GBI}$ ) using polymer helper and polyperoxidase-anti-mouse/rabbit IgG according to the manufacturer's instructions. Peroxidase substrate containing DAB ( $3,3^{\prime}$-diaminobenzidine) chromogen was added to the sections for 30 sec to develop the stain.

The overall staining intensity for BRCA 1 protein in cancer cells was scored on a 0 to 3 scale. A tissue section that did not exhibit any brown nuclear reactivity in any tumor cells was scored as 0 . When an occasional tumor cell showed positive nuclear staining but the total percentage of positivity in the tissue section was $<20 \%$, a score of 1 was given. A tissue section in which $20-80 \%$ of tumor cells exhibited positive nuclear staining was scored as 2 . More than $80 \%$ of tumor cells in a tissue section with positive nuclear staining was scored as $3^{29}$. In this study, score 0 was considered negative and 1-3 for positive.

Statistical analysis. The Pearson chi-square test and the Fisher's exact test were used to assess the association between categorical variables. Kaplan-Meier survival curves were employed to evaluate overall survival. Log-rank test was used to compare between survival curves. Multivariate regression analysis was performed using Cox proportional hazards model. To evaluate the independent effect of age at diagnosis on survival from breast cancer, a multivariate Cox regression model was performed by including all variables with a significant effect in the univariate analysis. $\mathrm{P}$ values $<$ 
Table 4 | Univariate and multivariate analysis of overall survival in young breast cancer patients

Univariate analysis

Multivariate analysis

\begin{tabular}{|c|c|c|c|c|}
\hline \multirow[b]{2}{*}{ Covariates } & & \multirow[b]{2}{*}{$\mathrm{HR}(95 \% \mathrm{Cl})$} & \multirow[b]{2}{*}{$P$} \\
\hline & $\mathrm{HR}(95 \% \mathrm{Cl})$ & $P$ & & \\
\hline No' & 1.000 & & \multicolumn{2}{|l|}{-} \\
\hline T1/T2 & 1.000 & & \multirow{2}{*}{$\begin{array}{c}1.000 \\
1.686(1.006-2.823)\end{array}$} & \\
\hline $\mathrm{T} 3 / \mathrm{T} 4$ & $3.219(2.009-5.159)$ & $<0.001$ & & 0.047 \\
\hline \multicolumn{5}{|l|}{ Node status } \\
\hline \multicolumn{5}{|l|}{ ER status } \\
\hline Negative & 1.000 & & \multirow{2}{*}{$\begin{array}{c}1.000 \\
0.485(0.290-0.811)\end{array}$} & \\
\hline Positive & $0.588(0.369-0.938)$ & 0.026 & & 0.006 \\
\hline \multicolumn{5}{|l|}{ BRCA 1 nuclear status } \\
\hline Negative & 1.000 & & \multirow{2}{*}{\multicolumn{2}{|c|}{-}} \\
\hline Positive & $0.851(0.309-2.342)$ & 0.851 & & \\
\hline
\end{tabular}

0.05 were considered to be statistically significant. All the analyses were conducted using SPSS version 19.0 statistical software.

Ethical approval. This study has been approved by the Ethics Committee of the Cancer Hospital of Shantou University Medical College, and was performed in accordance with the ethical standards laid down in the 1964 declaration of Helsinki and all subsequent revisions. All persons mentioned in the paper gave their informed consent prior to their inclusion in the study.

1. Siegel, R., Naishadham, D. \& Jemal, A. Cancer statistics, 2013. CA Cancer J Clin. 63, 11-30 (2013)

2. Zhou, P. \& Recht, A. Young age and outcome for women with early-stage invasive breast carcinoma. Cancer. 101, 1264-1274 (2004).

3. Xiong, Q. et al. Female patients with breast carcinoma age 30 years and younger have a poor prognosis: the M.D. Anderson Cancer Center experience. Cancer. 92, 2523-2528 (2001)

4. Sidoni, A., Cavaliere, A., Bellezza, G., Scheibel, M. \& Bucciarelli, E. Breast cancer in young women: clinicopathological features and biological specificity. Breast. 12, 247-250 (2003).

5. Han, W. et al. Young age: an independent risk factor for disease-free survival in women with operable breast cancer. BMC Cancer 4, 82 (2004).

6. Shavers, V. L., Harlan, L. C. \& Stevens, J. L. Racial/ethnic variation in clinical presentation, treatment, and survival among breast cancer patients under age 35 . Cancer. 97, 134-147 (2003).

7. Barber, M. D., Jack, W. \& Dixon, J. M. Diagnostic delay in breast cancer. Br J Surg. 91, 49-53 (2004)

8. Sariego, J. Breast cancer in the young patient. Am Surg. 76, 1397-1400 (2010).

9. Gajdos, C., Tartter, P. I., Bleiweiss, I. J., Bodian, C. \& Brower, S. T. Stage 0 to stage III breast cancer in young women. J Am Coll Surg. 190, 523-529 (2000).

10. Jmor, S. et al. Breast cancer in women aged 35 and under: prognosis and survival. J R Coll Surg Edinb. 47, 693-699 (2002).

11. Anders, C. K. et al. Young age at diagnosis correlates with worse prognosis and defines a subset of breast cancers with shared patterns of gene expression. J Clin Oncol. 26, 3324-3330 (2008).

12. Morrison, D. H., Rahardja, D., King, E., Peng, Y. \& Sarode, V. R. Tumour biomarker expression relative to age and molecular subtypes of invasive breast cancer. Br J Cancer. 107, 382-387 (2012).

13. Carvalho, F. M., Bacchi, L. M., Santos, P. P. \& Bacchi, C. E. Triple-negative breast carcinomas are a heterogeneous entity that differs between young and old patients. Clinics (Sao Paulo). 65, 1033-1036 (2010).

14. Bacchi, L. M., Corpa, M., Santos, P. P., Bacchi, C. E. \& Carvalho, F. M. Estrogen receptor-positive breast carcinomas in younger women are different from those of older women: a pathological and immunohistochemical study. Breast. 19, 137-141 (2010).

15. Kerlikowske, K. Epidemiology of ductal carcinoma in situ. J Natl Cancer Inst Monogr. 2010, 139-141 (2010).

16. Kroman, N. et al. Factors influencing the effect of age on prognosis in breast cancer: population based study. BMJ. 320, 474-478 (2000).

17. Oh, J. L. et al. The impact of young age on locoregional recurrence after doxorubicin-based breast conservation therapy in patients 40 years old or younger: How young is “young"? Int J Radiat Oncol Biol Phys. 65, 1345-1352 (2006)

18. Fourquet, A. et al. Prognostic factors of breast recurrence in the conservative management of early breast cancer: a 25-year follow-up. Int J Radiat Oncol Biol Phys 17, 719-25 (1989).
19. Elkhuizen, P. H. et al. Local recurrence after breast-conserving therapy for invasive breast cancer: high incidence in young patients and association with poor survival. Int J Radiat Oncol Biol Phys 40, 859-67 (1998).

20. Fowble, B. L. et al. The influence of young age on outcome in early stage breast cancer. Int J Radiat Oncol Biol Phys 30, 23-33 (1994).

21. Clarke, M. et al. Effects of radiotherapy and of differences in the extent of surgery for early breast cancer on local recurrence and 15-year survival: an overview of the randomised trials. Lancet 366, 2087-106 (2005).

22. de Bock, G. H. et al. Isolated loco-regional recurrence of breast cancer is more common in young patients and following breast conserving therapy: long-term results of European Organisation for Research and Treatment of Cancer studies. Eur J Cancer 42, 351-6 (2006).

23. Taghian, A. G. et al. Low locoregional recurrence rate among node-negative breast cancer patients with tumors $5 \mathrm{~cm}$ or larger treated by mastectomy, with or without adjuvant systemic therapy and without radiotherapy: results from five national surgical adjuvant breast and bowel project randomized clinical trials. J Clin Oncol 24, 3927-32 (2006).

24. Fredholm, H. et al. Breast cancer in young women: poor survival despite intensive treatment. PLoS One 4, e7695 (2009).

25. Zhang, J. \& Powell, S. N. The role of the BRCA1 tumor suppressor in DNA doublestrand break repair. Mol Cancer Res 3, 531-9 (2005).

26. Armes, J. E. et al. Distinct molecular pathogeneses of early-onset breast cancers in BRCA1 and BRCA2 mutation carriers: a population-based study. Cancer Res 59, 2011-7 (1999).

27. Peto, J. et al. Prevalence of BRCA1 and BRCA2 gene mutations in patients with early-onset breast cancer. J Natl Cancer Inst 91, 943-9 (1999).

28. Malone, K. E. et al. Frequency of BRCA1/BRCA2 mutations in a population-based sample of young breast carcinoma cases. Cancer 88, 1393-402 (2000).

29. Yoshikawa, K. et al. Reduction of BRCA1 protein expression in Japanese sporadic breast carcinomas and its frequent loss in BRCA1-associated cases. Clin Cancer Res 5, 1249-61 (1999).

30. Rakha, E. A. et al. Expression of BRCA1 protein in breast cancer and its prognostic significance. Hum Pathol. 39, 857-865 (2008).

31. Xiang, T. et al. Targeting the Akt/mTOR pathway in Brcal-deficient cancers. Oncogene. 30, 2443-2450 (2011).

32. Bogdani, M. et al. Loss of nuclear BRCA1 localization in breast carcinoma is age dependent. Virchows Arch. 440, 274-279 (2002).

33. Kim, D., Jung, W. Koo, J. S. The expression of ERCC1, RRM1, and BRCA1 in breast cancer according to the immunohistochemical phenotypes. J Korean Med Sci 26, 352-9 (2011).

34. Yang, Q. et al. Prognostic significance of BRCA1 expression in Japanese sporadic breast carcinomas. Cancer. 92, 54-60 (2001).

35. Guler, G. et al. Aberrant expression of DNA damage response proteins is associated with breast cancer subtype and clinical features. Breast Cancer Res Treat. 129, 421-432 (2011).

36. Nakai, K. et al. Predictive value of MGMT, hMLH1, hMSH2 and BRCA1 protein expression for pathological complete response to neoadjuvant chemotherapy in basal-like breast cancer patients. Cancer Chemother Pharmacol. 69, 923-930 (2012).

\section{Acknowledgments}

This work is partly supported by the funds from Major State Basic Research Development Program of China (No.2011CB707705); Natural Science Foundation Committee (NO.31271068), and Guangdong Provincial Key Laboratory of Breast Cancer Diagnosis 
and Treatment to Dr. Guo-Jun Zhang, and Shantou Manicipal Sci-Tech Bureau (2013-88) to Si-Qi Qiu.

\section{Author contributions}

J.T.W. and W.H.H. collected patients' clinical records, performed experiments, prepared the figures and wrote the manuscript. S.Q.Q., X.L.W. and J.L. contributed to the pathology studies. C.W.D. collected patient information. G.J.Z. designed the study and wrote the paper. J.T.W. and W.H.H. contributed equally to the work. All authors reviewed the manuscript.

\section{Additional information}

Competing financial interests: The authors declare no competing financial interests.

How to cite this article: Wei, J.-T. et al. Clinicopathological features and prognostic factors of young breast cancers in Eastern Guangdong of China. Sci. Rep. 4, 5360; DOI:10.1038/ srep05360 (2014)

(c) (i) (5) This work is licensed under a Creative Commons Attribution-NonCommercialNoDerivs 4.0 International License. The images or other third party material in this article are included in the article's Creative Commons license, unless indicated otherwise in the credit line; if the material is not included under the Creative Commons license, users will need to obtain permission from the license holder in order to reproduce the material. To view a copy of this license, visit http:// creativecommons.org/licenses/by-nc-nd/4.0/ 